\title{
Utilidad de la morfología de los huevos como un método indirecto para identificar Anopheles benarrochi Gabaldón, Cova García \& López, Anopheles oswaldoi (Peryassu) y Anopheles rangeli Gabaldón, Cova García \& López, (Diptera: Culicidae) en Putumayo, Colombia
}

\begin{abstract}
Dora Amparo Estrada ${ }^{1}$, Martha L. Quiñones ${ }^{1}$, Diana Maria Sierra ${ }^{1}$, David A. Calle ${ }^{1}$, Fredy Ruiz ${ }^{1}$, Holmes F. Erazo ${ }^{2}$, Yvonne-Marie Linton ${ }^{3}$

${ }^{1}$ Programa de Estudio y Control de Enfermedades Tropicales, PECET, Facultad de Medicina, Universidad de Antioquia, Medellín, Colombia.

2 Departamento Administrativo de Salud, DASALUD, Putumayo, Colombia.

3 The Mosquitoes Programme and Biomedical Sciences Theme, Department of Entomology, The Natural History Museum, London, England.

La identificación correcta de las hembras es esencial para el éxito de cualquier estudio de epidemiología, resistencia a insecticidas o de control de vectores. En el departamento del Putumayo, en el sur de Colombia, la transmisión de malaria continúa siendo un problema, a pesar de la ausencia de los vectores principales de Latinoamérica (Anopheles darlingi Root, Anopheles nuneztovari Gabaldón, Anopheles albimanus Wideman, Anopheles trinkae Faran) en esta región. Se recolectaron. con cebo humano, hembras de Anopheles y se encontró una variante morfológica de Anopheles benarrochi, que en su estadio adulto fácilmente se confunde con Anopheles oswaldoi. La identificación de hembras de Anopheles, particularmente del subgénero Nyssorhynchus, es en general notoriamente difícil debido a la superposición de caracteres morfológicos en el estadio adulto; por tanto, las colecciones deben estar ligadas a la cría de material asociado para identificar correctamente las especies. Esto requiere tiempo y es difícil de obtener en muchas ocasiones. Se presenta un método indirecto de identificación de las especies $A$. benarrochi, $A$. oswaldoi y Anopheles rangeli del sur de Colombia usando la morfología de los huevos de hembras silvestres. Los huevos de $A$. rangeli y $A$. benarrochi se diferencian por la corona anterior, la cual es apical en $A$. rangeli y con paredes altas, mientras que en $A$. benarrochi es ventral y con paredes más cortas. Esta corona está ausente en $A$. oswaldoi. Estas diferencias fueron obvias incluso bajo un microscopio de luz, lo que hace posible una identificación correcta de estas especies en condiciones de campo. Se muestra cómo la observación de la morfología de los huevos puede permitir la determinación taxonómica correcta, aunque indirecta, de estas tres especies de Nyssorhynchus encontradas en el sur de Colombia, el cual puede ser útil también en otras regiones de Latinoamérica, en donde se encuentre la variante morfológica de $A$. benarrochi en simpatría con $A$. oswaldoi.
\end{abstract}

Palabras clave: Nyssorhynchus, huevos, Anopheles, Colombia, microscopia electrónica.

Egg morphology as an indirect method to identify Anopheles benarrochi, Anopheles oswaldoi and Anopheles rangeli (Diptera: Culicidae)

In the Department of Putumayo in southern Colombia, malaria transmission has continued in the absence of the 4 traditional Latin American vector species - Anopheles darlingi, Anopheles nuneztovari, Anopheles albimanus or Anopheles trinkae. Human bait collections yielded Anopheles mosquitoes and a morphological variant of Anopheles benarrochi, the adult females of which can easily be misidentified as Anopheles oswaldoi. Species identification of females of Anopheles in the subgenus Nyssorhynchus is generally difficult due to overlapping morphological characters; therefore, progeny of field collected females were link-reared to assess species identity. Herein a robust method is presented to identify the species Anopheles 
benarrochi, Anopheles oswaldoi and Anopheles rangeli from southern Colombia, using the morphology of the eggs induced from wild-caught females. Eggs of $A$. rangeli and $A$. benarrochi were differentiated on the basis of the anterior crown. In $A$. rangeli, this feature is positioned apically with high walls. In $A$. benarrochi, anterior crown is positioned more ventrally with comparatively shorter walls. No crown is present in $A$. oswaldoi. These differences are clear with the aid of a dissecting microscope and make accurate species determination possible even in field conditions. Egg morphology is shown to be an accurate, albeit indirect, method for the taxonomic determination for the three southern Colombian species and may also be useful in other regions of Latin America where the morphological variant of $A$. benarrochi is sympatric with A. oswaldoi.

Key words: Nyssorhynchus, eggs, Anopheles, Colombia, scanning electron microscopy.

Los estudios más completos sobre los mosquitos Anopheles del Neotrópico pertenecientes al subgénero Nyssorhynchus se reportan en Faran (1), Faran \& Linthicum (2) y Linthicum (3). Estas publicaciones contienen las claves taxonómicas más usadas. Algunas especies de este subgénero muestran una variación intraespecífica y superposición interespecífica de caracteres morfológicos en el estadio adulto, principalmente hembras, y en la pupa, que hacen difícil su identificación usando estas claves y se pueden producir identificaciones erróneas (4). Faran (1) menciona que las genitalias masculinas y la quetotaxia de las larvas ofrecen las características más confiables para una correcta determinación de estas especies. Por esta razón, es importante basar la identificación de especies en estados de vida asociados, pero estas crías demandan tiempo y algunas veces son difíciles de realizar en condiciones de campo.

En estudios epidemiológicos y de control de vectores es indispensable realizar la identificación de una forma rápida y correcta, de hembras de mosquitos antropofílicos. Es justamente en este estadio en el que se hace difícil una correcta identificación, principalmente de hembras del subgénero Nyssorhynchus. A pesar de que muchas especies se han identificado con éxito utilizando la morfología de sus huevos, incluyendo miembros de complejos de especies como el

\footnotetext{
Correspondencia:

Martha L. Quiñones, Programa de Estudio y Control de Enfermedades Tropicales, Facultad de Medicina, Universidad de Antioquia, apartado aéreo 1226, Medellín, Colombia. Fax: (+574) 5716675. martqp@yahoo.com
}

Recibido: 09/05/03; aceptado: 12/09/03 complejo Anopheles maculipennis Meigen (5), los estudios de Faran (1), Faran \& Linthicum (2) y Linthicum (3) no involucran los caracteres de los huevos como herramientas para la identificación de especies. La observación de la morfología de los huevos de hembras silvestres que ovipositan en el laboratorio, junto con la morfología de las madres, podrían ser herramientas útiles para una correcta identificación de especies de Nyssorhynchus.

El potencial de la microscopia electrónica de barrido (MEB) para elucidar caracteres diagnóstico en huevos de Anopheles fue reconocido por Hinton (6). La MEB se ha convertido, cada vez con mayor frecuencia, en una herramienta importante al generar información de las estructuras de los huevos. Los estudios detallados de las células coriónicas, densidad y estructura de los tubérculos de la superficie ventral y detalles de los flotadores por medio de la MEB permiten el análisis de características ultrastructurales para poder hacer comparaciones entre especies y poblaciones (7). Utilizando la MEB, se han descrito los huevos de 12 de las 16 especies de la Sección Albimanus del subgénero Nyssorhynchus (8), estas especies son: Anopheles albimanus Wiedemann (9); Anopheles aquasalis Curry $(10,11)$, Anopheles benarrochi Gabaldón, Cova García \& López, Anopheles dunhami Causey, Anopheles konderi Galvao \& Damasceno, Anopheles oswaldoi (Peryassu), Anopheles nuneztovari Gabaldón (12); Anopheles rangeli Gabaldón, Cova Garcia \& Lopez y Anopheles trinkae Faran (como A. dunhami)(13); Anopheles rondoni (Neiva \& Pinto) (14); Anopheles strodei Root, Anopheles triannulatus Neiva \& Pinto (7), Anopheles galvaoi Causey, Deane \& Deane y Anopheles evansae (Brethes) (15). 
Faran (1) y Faran \& Linthicum (2) reportaron $A$. evansae con una distribución restringida a Brasil, Bolivia y Argentina. Sin embargo, hay reportes de $A$. evansae en varias localidades de Colombia recolectados en cebo humano o en trampa Shannon (16-20). En el departamento del Putumayo, $A$. evansae y $A$. rangeli se han reportado como las especies más antropofílicas (21), junto con A. oswaldoi (22). Usando las claves de Faran (1) y Faran \& Linthicum (2), algunos adultos hembras de Putumayo concuerdan con las descripciones de adultos hembras de $A$. evansae, pero difieren en otros estadios (4). En esta región se encontró una variante morfológica de $A$. benarrochi, la cual en su estadio adulto es similar a las descripciones de las hembras de $A$. evansae y probablemente los registros de $A$. evansae de Putumayo corresponden realmente a $A$. benarrochi. Algunos ejemplares de $A$. benarrochi en Putumayo se pueden confundir tambien con $A$. oswaldoi (4). La identidad de los especímenes identificados como $A$. evansae procedente de otras áreas de Colombia generan dudas ya que podrían corresponder también a esta variante morfológica de $A$. benarrochi.

En una revisión llevada a cabo por Ferro (16) y en reportes internos del antiguo Servicio de Erradicación de Malaria y unidades locales de salud de Putumayo, A. evansae (como Anopheles noroestensis) se reporta como la especie responsable de transmisión de malaria en Putumayo. Sin embargo, esto se ha basado solamente en su alta frecuencia de picaduras a humanos, sin evidencia de su infectividad natural con Plasmodium. Los vectores tradicionales de malaria en Latinoamérica no se han reportado en Putumayo. A. darlingi sólo se registra en el municipio de Puerto Leguízamo. A. trinkae, quien se ha incriminado como vector en un área vecina al Putumayo, en Perú (23), no se ha reportado en Putumayo. La presencia de $A$. nuneztovari se reporta en 1960, pero no hay reportes después de ese año. $A$. nuneztovari es un vector reconocido de malaria en Colombia (24), Perú (23) y Venezuela $(25,26)$. Dadas las dificultades de diferenciar hembras adultas de Nyssorhynchus (1), particularmente entre $A$. trinkae, $A$. rangeli, $A$. oswaldoi, A. evansae, A. nuneztovari, especial- mente, el morfotipo II descrito en Venezuela (27), y la variante morfológica de $A$. benarrochi, se podría pensar que especies vectoras como $A$. nuneztovario $A$. trinkae pudieran estar presentes en Putumayo y debido a la dificultad de discriminar las especies del subgénero Nysshorhynchus a partir de hembras, estuvieran erróneamente identificadas. Para incriminar las especies responsables de la transmisión en Putumayo es esencial primero identificar correctamente las especies antropofílicas de la región.

Este estudio se realizó para evaluar si las características de los huevos podían ser utilizadas para facilitar una identificación acertada de hembras silvestres de las especies $A$. benarrochi y $A$. oswaldoi, principalmente. Se incluyó tambien $A$. rangeli por hacer parte de las especies con más antropofília en Putumayo.

\section{Metodología}

Se recolectaron hembras de Anopheles con cebo humano en las localidades de Lisberia y La Manuela del municipio de Puerto Asís, departamento de Putumayo, entre febrero de 1999 y julio de 2001. Las hembras se alimentaron con sangre colocándolas sobre ratón o curí y se transportaron vivas al Laboratorio de Entomología del Programa de Estudio y Control de Enfermedades Tropicales (PECET) de la Universidad de Antioquia en vasos de icopor. Sobre cada vaso se colocó una solución de glucosa al $5 \%$ y se mantuvieron en el insectario por 203 días antes de inducir la oviposición. Para la inducción de la postura, se procedió a adormecer cada hembra colocándola dentro de un frasco que contenía en el fondo una mota de algodón con acetato de etilo. Cada hembra se colocó sobre agua destilada en un pequeño vaso de plástico después de remover con pinzas un ala y una pata posterior. Cuarenta y ocho horas después de la oviposición, se colocaron aproximadamente 20 huevos por hembra en glutaraldehído al $2 \%$ y se guardaron a $5^{\circ} \mathrm{C}$. El resto de los huevos de cada vaso fue criado hasta adulto. Las pieles asociadas de larva y pupa se guardaron en etanol al $80 \%$ y el respectivo adulto se montó en alfiler para identificación taxonómica. También se realizaron montajes de genitalias masculinas. La identificación de la 
especie se realizó observando la morfología de los huevos y se confirmó examinando las pieles de larva, pupa, adulto y genitalia masculina de la progenie de la misma familia, según las descripciones y clave de Faran (1). Además, se realizó una determinación de especies de cada familia basado en similaridades de las secuencias de ITS2 con secuencias disponibles en el GenBank (datos no mostrados).

Para microscopia electrónica y obtención de medidas detalladas de las características de los huevos se seleccionaron muestras de 5 familias de cada especie, con la excepción de $A$. oswaldoi de los cuales sólo dos familias se encontraron en condiciones lo suficientemente buenas para ser fotografiadas. Los huevos destinados para fotografía se deshidrataron en gradientes de alcohol de $30 \%$ al $100 \%$, se llevaron a secado de punto crítico con $\mathrm{CO}_{2}$ y se recubrieron con oropaladio. Los huevos se observaron en un microscopio electrónico Hitachi S-510 del Instituto de Biología de la Universidad de Antioquia. Algunas muestras adicionales se procesaron y examinaron en el Museo de Historia Natural de Londres.

\section{Resultados}

Con base en la morfología de los huevos se identificaron tres especies: $A$. rangeli (38 familias), A. oswaldoi (35 familias) y $A$. benarrochi (268 familias). Estas tres especies presentan huevos con características tan diferentes entre ellas, que facilitaron notablemente la identificación, principalmente entre $A$. benarrochiy $A$. oswaldoi. Para la observación de las características de los huevos fue suficiente un microscopio de luz, lo que es de gran importancia y utilidad práctica. Es necesario resaltar que fue necesario iluminar el campo por encima, colocando una lámpara con la luz hacia el portaobjetos y disminuir la intensidad de la luz en la base del microscopio.

Las características por las que se pudieron diferenciar los huevos de estas tres especies fueron: en $A$. rangeli una característica diagnóstico es la presencia de una corona circular con paredes altas en la terminación anterior del huevo. La morfología de los huevos de $A$. rangeli de Putumayo (figura 1A) coincide con las descripciones para esta especie de Linley \& Lounibos (13).

Los huevos de $A$. benarrochi (figura 1B) presentan similarmente una corona circular, pero en posición ventral y de paredes más cortas que los huevos de $A$. rangeli. Esta característica junto con la forma de las células dorsales, las cuales se muestran agrupadas y perforadas con poros, distinguen a $A$. benarrochi de otras especies de Nyssorhynchus. La morfología de los huevos de $A$. benarrochi coincide con las descripciones de Lounibos et al. (7).

Los huevos de $A$. oswaldoi se caracterizan por presentar un borde pequeño a lo largo de la parte interna de los flotadores como se muestra en la figura $1 \mathrm{C}$, no presentan corona, característica que permite diferenciar $A$. oswaldoi de $A$. rangeli y $A$. benarrochi. Los huevos de $A$. oswaldoicoincidieron con la descripción de Lounibos et al. (7).

En el cuadro 1 se muestran algunas mediciones de los huevos, en donde se evidencia la ausencia de corona de $A$. oswaldoi, que permite diferenciar los huevos de $A$. oswaldoi de los huevos de $A$. rangeli y $A$. benarrochi. Entre estas dos últimas especies, tanto el ancho como la altura de la pared de la corona anterior permiten su separación.

\section{Discusión}

En estudios epidemiológicos o en evaluaciones de métodos de control de malaria, es necesario identificar las especies de Anopheles correctamente. En situaciones en donde la identificación de hembras de Anopheles no es fácil, como es el caso de algunas de las especies del subgénero Nyssorhynchus, esta identificación puede realizarse indirectamente permitiendo que las hembras realicen su ovipostura y examinando la morfología de los huevos. Este estudio muestra que las especies $A$. rangeli, $A$. oswaldoi y la variante morfológica de $A$. benarrochien el sur de Colombia (4) se pueden identificar claramente basados en la morfología de los huevos. Este método puede ser muy útil debido a la gran variabilidad interespecifica y superposición de caracteres entre $A$. benarrochi y $A$. oswaldoi en sus estadios adultos (4). El personal de las unidades locales de salud de entomología puede 


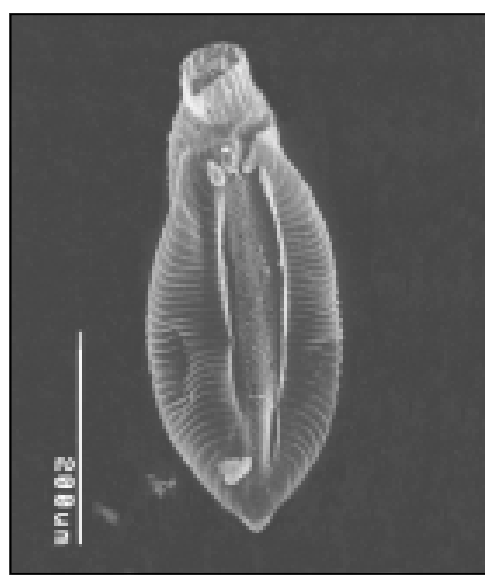

A) Anopheles rangeli

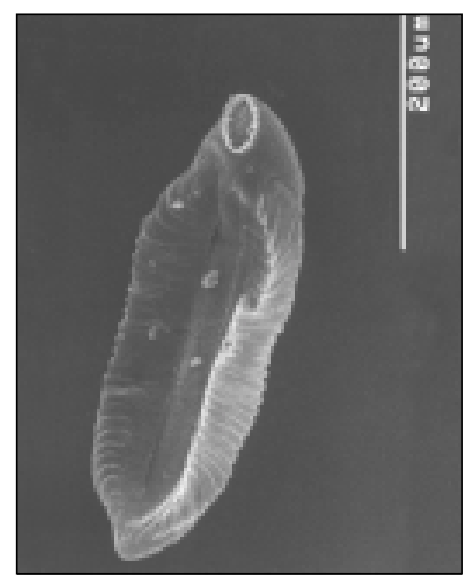

B) Anopheles benarrochi

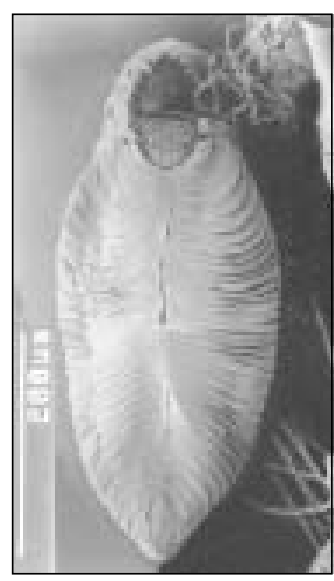

C) Anopheles oswaldoi

Figura 1. Huevos de: A) Anopheles rangeli, B) Anopheles benarrochi y C) Anopheles oswaldoi de hembras recolectadas picando humanos en el departamento de Putumayo, Colombia.

Cuadro 1. Comparación de los promedios y rangos de las características de los huevos de Anopheles rangeli, Anopheles benarrochi y Anopheles oswaldoi. Medidas en micras $(\mu)$.

\begin{tabular}{lcccccc}
\hline Características & $\mathbf{n}$ & $\boldsymbol{A}$. rangeli & $\mathbf{n}$ & $\boldsymbol{A}$. benarrochi & $\mathbf{n}$ & $\boldsymbol{A}$. oswaldoi \\
\hline Longitud (L) & 95 & $476,90 \pm 2,40$ & 80 & $463,30 \pm 2,60$ & 2 & $404,70 \pm 18,7$ \\
Ancho promedio (A) & 95 & $147,70 \pm 1,60$ & 80 & $138,60 \pm 1,75$ & 2 & $158,80 \pm 19,2$ \\
Razón L/A & 95 & $3,30 \pm 0,05$ & 80 & $3,40 \pm 0,05$ & 2 & $2,50 \pm 0,19$ \\
Ancho anterior & 93 & $138,80 \pm 2,50$ & 80 & $130,10 \pm 2,70$ & 1 & 128,40 \\
Ancho posterior & 93 & $53,10 \pm 1,60$ & 80 & $51,40 \pm 1,70$ & 1 & 41,20 \\
Longitud de los flotadores & 93 & $358,50 \pm 4,80$ & 80 & $358,10 \pm 5,30$ & 2 & $340,80 \pm 1,1$ \\
\% de los flotadores & 92 & $75,40 \pm 0,90$ & 80 & $77,30 \pm 1,10$ & 2 & $84,40 \pm 4,1$ \\
No. surcos en los flotadores & 92 & $35,20 \pm 0,30$ & 77 & $32,80 \pm 0,40$ & 2 & $35,00 \pm 1$ \\
$\begin{array}{l}\text { Ancho de los surcos } \\
\text { Distancia entre los flotadores }\end{array} \quad 92$ & $10,60 \pm 0,10$ & 77 & $10,90 \pm 0,10$ & 2 & $9,70 \pm 0,03$ \\
$\quad$ y la corona anterior & 84 & $40,40 \pm 1,70$ & 64 & $40,70 \pm 1,90$ & & NA \\
$\begin{array}{l}\text { Ancho de la corona anterior } \\
\text { Altura de la pared de la }\end{array}$ & 90 & $52,00 \pm 1,40$ & 68 & $35,10 \pm 1,60$ & $\mathrm{NA}$ \\
$\quad$ corona anterior & 92 & $36,20 \pm 0,60$ & 68 & $15,50 \pm 0,70$ & & $\mathrm{NA}$ \\
\hline
\end{tabular}

(NA: no aplica por no presentar corona anterior)

implementar las colecciones de mosquitos y la posterior obtención de sus huevos, particularmente en regiones en donde se reporta la presencia de $A$. evansae, $A$. oswaldoi o $A$. benarrochi para corroborar las determinaciones realizadas basadas solamente en la morfología de hembras adultas. La posibilidad de observar las características en los huevos por medio de un microscopio de luz facilita una identificación correcta de estas especies en condiciones de campo. Aunque se han publicado pocas claves para huevos de Anopheles, las existentes proveen suficientes ilustraciones y descripciones para permitir su uso por funcionarios de salud en campo $(6,28)$. Sería deseable, sin embargo, elaborar una clave para huevos de Anopheles de Colombia.

En algunas especies del subgénero Nyssorhynchus, la morfología de los huevos no permite diferenciar entre especies, debido a que algunas especies presentan un alto nivel de variabilidad morfológica en este estadio, o los huevos presentan similaridades con los de otras especies bajo el microscopio de luz. Por ejemplo, para la especie $A$. strodei, se ha reportado la existencia de variación intraespecifica (29) en la morfología de los huevos, y huevos de varias especies pueden 
ser indistinguibles al observarlos en un estereoscopio o microscopio de luz, como son las especies $A$. nuneztovari y $A$. albimanus, los cuales presentan una morfología similar, o $A$. oswaldoi y A. konderi, entre otras. Por esta razón, Delgado \& Rubio-Palis (27) expresan la poca confiabilidad al usar solamente características de los huevos para identificación de especies. Sin embargo, en este estudio la observación de los huevos permitió realizar una identificación rápida y corroborar las identificaciones de las hembras aisladas para cría de isofamilias de las especies A. rangeli, $A$. oswaldoi y $A$. benarrochi de Putumayo. La morfología de los huevos, junto con las características de sus estadios asociados: larva, pupa, genitalia masculina y adulto, permitieron descartar la presencia de otras especies de Nyssorhynchus, de las cuales se sospechaba o se había mencionado su presencia en esta región en reportes internos de DASALUD Putumayo entre las que se cuenta $A$. nuneztovari, A. trinkae y $A$. evansae. Cabe anotar que en 3 años de recolecciones no se encontró evidencia de su presencia en Putumayo. Estas especies presentan características en todos sus estadios que las diferenciarían de $A$. benarrochi, $A$. oswaldoi y $A$. rangeli $(1,2)$. Particularmente en los huevos, las características más notorias y visibles bajo el microscopio para diferenciar $A$. trinkae de las especies antes mencionadas son el tamaño de los flotadores; en $A$. trinkae son más grandes que en $A$. rangeli, la presencia de una corona, similar a la de $A$. rangeli, pero sobresaliendo el ápice del huevo por el centro de la corona. Una descripción detallada de la morfología de los huevos de $A$. rangeli y $A$. trinkae (como $A$. dunhami) la realizaron Linley y Lounibos (13). $A$. nuneztovari se podría confundir en su estadio adulto con $A$. trinkae, $A$. benarrochi o $A$. rangeli $(1,2)$; sin embargo, sus huevos presentan diferencias que permiten una fácil separación como es la ausencia de la corona en los huevos de $A$. nuneztovarimientras que $A$. trinkae, $A$. benarrochi y $A$. rangeli presentan esta corona. Una completa descripción de los huevos de $A$. nuneztovari la realizó Linley et al. (12). Aunque se ha descrito una variabilidad en las células coriónicas en huevos de $A$. nuneztovari, esta variabilidad es notable sólo bajo microscopia electrónica (12). Los adultos de
A. evansae se pueden confundir con la variante de $A$. benarrochi encontrada en Putumayo, similarmente los huevos de estas dos especies permiten su fácil separación; la diferencia más evidente es la presencia de la corona en $A$. benarrochi y su ausencia en $A$. evansae, entre otras diferencias en los estadios inmaduros y genitalia masculina. Una reciente descripción de los huevos de $A$. evansae la realizó Salum et al. (15).

$A$. benarrochi se menciona con una amplia distribución no sólo en Colombia, sino también en países vecinos como Perú y Brasil $(1,30)$. En Colombia se registra principalmente en la región de la Orinoquia de los Llanos Orientales de Colombia $(1,31)$. Sin embargo, esta especie no se menciona en reportes locales recientes de las diferentes secretarias de salud. Según las descripciones de Faran (1), las hembras adultas de $A$. benarrochi son muy similares a las de $A$. aquasalis y $A$. strodei. Inclusive en la descripción original de esta especie Gabaldón et al. (32) mencionan que los adultos hembras son imposibles de distinguir de $A$. strodei, $A$. nuneztovari o $A$. rangeli. El hallazgo de una variante morfológica de $A$. benarrochi que hace que sus hembras sean indistinguibles de $A$. oswaldoi en Putumayo, utilizando las claves disponibles, fue una observación interesante que dificulta aún más su determinación taxonómica a partir de hembras. Al parecer, las claves en uso para el subgénero Nyssorhynchus $(1,2,33)$ no permiten una correcta identificación de los adultoshembras de esta especie en el sur de Colombia, a menos que se disponga de estadios asociados. Debido a esta dificultad con las claves, es probable que $A$. benarrochi esté siendo incorrectamente identificada en otras áreas del país y países vecinos. La identificación de $A$. benarrochi en Putumayo se realizó con base en la morfología de los huevos y las características de sus larvas, pupas y genitalias masculinas (4) así como por secuencias de ADN (datos no mostrados) de progenies de isofamilias. Sin embargo, es difícil realizar todas estas actividades en las diferentes regiones del país y es necesario disponer de una alternativa más fácil para la correcta identificación de las especies, particularmente en regiones donde se reporta la presencia de $A$. evansae y $A$. 
oswaldoi, los cuales podrían corresponder a $A$. benarrochi.

En este trabajo se muestra la utilidad de usar la morfología de los huevos, obtenidos a partir de la recolección de hembras silvestres individualizadas, como un método relativamente sencillo, el cual puede ser realizado en el campo y que permite identificar o confirmar la identificación de algunas especies de Anopheles en su estadio adulto, particularmente para identificar $A$. benarrochi en regiones donde se registra $A$. evansae o $A$. oswaldoi. Este método puede permitir una determinación taxonómica correcta, aunque indirecta, de $A$. benarrochi, $A$. oswaldoi y A. rangeli en el sur de Colombia, el cual puede ser útil también en otras regiones de Latinoamérica, en donde se encuentre la variante morfológica de $A$. benarrochi en simpatría con $A$. oswaldoi.

\section{Agradecimientos}

Este estudio fue financiado por The Wellcome Trust, Colciencias (Cod. 1115-04-460-98) y el CODI de la Universidad de Antioquia. Expresamos nuestros agradecimientos a Albeiro Acevedo del Instituto de Biología de la Universidad de Antioquia por la colaboración en el procesamiento de las muestras para MEB y las fotografías, a Theresa Howard del Museo de Historia Natural (NHM) de Londres por su asistencia con las fotografías por MEB de A. oswaldoi y a Ralph Harbach (NHM) por la invaluable ayuda en la preparación y edición del manuscrito.

\section{Referencias}

1. Faran ME. Mosquito studies (Diptera: Culicidae) XXXIV. A revision of the Albimanus section of the subgenus Nyssorhynchus of Anopheles. Contr Am Entom Inst 1980;15:1-214

2. Faran ME, Linthicum KJ. A handbook of the Amazonian species of Anopheles (Nyssorhynchus) (Diptera: Culicidae). Mosq Syst 1981;13:1-18.

3. Linthicum KJ. A revision of the Argyritarsis Section of the Subgenus Nyssorhynchus of Anopheles (Diptera: Culicidae). Mosq Syst 1988;20:98-270.

4. Quiñones ML, Harbach RE, Calle DA, Ruiz F, Erazo HF, Linton Y-M. Variante morfológica de adultos hembras de Anopheles benarrochi (Diptera: Culicidae) en Putumayo, Colombia. Biomédica 2001;21:351-9.

5. White GB. Systematic reappraisal of the Anopheles maculipennis complex. Mosq Syst 1978;10:13-44.
6. Hinton HE. Observations on the biology and taxonomy of the eggs of Anopheles mosquitoes. Bull Ent Res 1968;57:495-508.

7. Lounibos LP, Duzak D, Linley JR. Comparative egg morphology of six species of the Albimanus section of Anopheles (Nyssorhynchus) (Diptera: Culicidae). J Med Ent 1997;34:136-55.

8. Harbach RE. Review of the internal classification of the genus Anopheles (Diptera: Culicidae): the foundation for comparative systematics and phylogenetic research. Bull Ent Res 1994;84:331-42.

9. Rodríguez MH, Chávez B, Orozco A, Loyola EG, Martínez-Palomo A. Scanning electron microscopic observations of Anopheles albimanus (Diptera: Culicidae) eggs. J Med Ent 1992;29:400-6.

10. Valle D, Monnerat AT, Soares MJ, Rosa-Freitas MG, Pelajo-Machado M, Vale BS, Lenzi HL, Galler R, Lima JBP. Mosquito embryos and eggs: polarity and terminology of chorionic layers. J Insect Physiol 1999;45: 701-8.

11. Maldonado V, Finol HJ, Navarro JC. A. aquasalis eggs from two localities compared by scanning electron microscopy. Mem Inst Oswaldo Cruz 1997;92:48791.

12. Linley JR, Lounibos LP, Conn J, Duzak D, Nishimura N. A description and morphometric comparison of eggs from eight geographic populations of the South American malaria vector Anopheles (Nyssorhynchus) nuneztovari (Diptera: Culicidae). J Am Mosq Control Assoc 1996;12:275-92.

13. Linley JR, Lounibos LP. The eggs of Anopheles (Nyssorhynchus) rangeli and Anopheles (Nyssorhynchus) dunhami (Diptera: Culicidae). Mosq Syst 1993;25:157-69.

14. Foratinni OP, Sallum AM, Bergo ES, Flores DC. Ultrastructure of eggs of Anopheles rondoni, Anopheles lutzii and Anopheles parvus, three species of the subgenus Nyssorhynchus. J Am Mosq Control Assoc 1998:4:256-65.

15. Sallum MA, Bergo ES, Forattini OP, Flores DC. The eggs of Anopheles galvaoi and Anopheles evansae, two species of the subgenus Nyssorhynchus. J Am Mosq Control Assoc 2002;18:10-15.

16. Ferro CA. Revisión de los recursos aplicables a la lucha contra el Paludismo. Rev Esc Nac Salud Pública 1979;5:11-8.

17. Quiñones ML, Suárez MF, Fleming GA. Distribución y bionomía de los anofelinos de la Costa Pacífica de Colombia. Col Médica 1987;18:19-24.

18. Quiñones ML, Villarreal LI, Suárez MF. Distribution and biting behaviour of Anopheles species in Casanare, Colombia. Am Mosq Control Assoc 1992;8:305-6.

19. Herrera S, Suárez MF, Sánchez GI, Quiñones ML, Herrera M. Uso de la técnica inmuno-radiometrica 
(IRMA) en Anopheles de Colombia para la identificación de esporozoítos de Plasmodium. Col Médica 1987; 18:2-6.

20. Pérez L, Suárez M, Murcia L, de la $\mathrm{Hoz} F$, Olano VA, Brochero H, Toro P. La malaria en el Amazonas: conocimientos, practicas, prevalencia de parasitemia y evaluación entomológica en mayo de 1997. Biomédica 1999;19:93-102.

21. Suárez MF, Quiñones ML, Wirtz RA. Anopheles rangeli: a suspected vector of Plasmodium vivax in southern Colombia. The $39^{\text {th }}$ Annual Meeting of the American Society of Tropical Medicine and Hygiene, New Orleans; 1999. p.158.

22. Quiñones ML, Linton Y-M, Harbach RE, Estrada DA, Erazo HF, Calle DA, Ruiz JF. Malaria vector species in southern Colombia: species determination and natural infectivity. XVth International Congress for Tropical Medicine and Malaria. 20-25 August, Cartagena de India, Colombia; 2000. p.108.

23. Hayes J, Calderon G, Falcon R, Zambrano V. Newly incriminated Anopheles vectors of human malaria parasites in Junin Department, Peru. J Am Mosq Control Assoc 1987;33:418-22.

24. Elliot R. The influence of vector behaviour on malaria transmission. Am J Trop Med Hyg 1972;21:755-63.

25. Pintos P, Sabril López V. Esporozoítos en Anopheles ( $N$.) nuñeztovari en área de malaria refractaria. Bol Dir Malariol San Amb 1968;88:375-81.

26. Rubio-Palis Y, Curtis CF. Biting and resting behaviour of anophelines in western Venezuela and implications for control of malaria transmission. Med Vet Entomol 1992;6:375-81.

27. Delgado N, Rubio-Palis Y. Morphometric characterisation of the malaria vector Anopheles nuñeztovari (Diptera: Culicidae) from Western Venezuela. Mosq Syst 1992;24:231-41.

28. Causey OR, Deane LM, Deane, MP. An illustrated key to the eggs of thirty species of Brazilian anophelines with several new descriptions. Am J Hyg 1944;39:1-7.

29. Cova Garcia P, Sutil E. Claves gráficas para la clasificación de anofelinos de Venezuela. Caracas: División de Endemias Rurales, Dirección de Malariología y Saneamiento Ambiental, Ministerio de Sanidad y Asistencia Social; 1977. p.1-92.

30. Sallum MAM, Bergo ES, Forattini OP. First record of Anopheles benarrochi Gabaldón, Cova Garcia \& Lopez from the State of Sao Paulo, Southern Brazil. Mem Inst Oswaldo Cruz 1997;92:233-4.

31. Rengifo S, de Zulueta J. Five years' observations of rural malaria in eastern Colombia. Am J Trop Med Hyg 1952;1:598-611.

32. Gabaldón A, Cova-García P, López JA. Anopheles (Nyssorhynchus) benarrochi, una nueva especie de la subserie Triannulatus. Publ Div Malariol 1941;7: 3-24.

33. Suárez MF, Quiñones M, Fleming GA Robayo M. Guía introductoria a la morfología de Anopheles y clave para determinación de las principales especies de Colombia. Bogotá, Colombia: Dirección de Campañas Directas, Ministerio de Salud; 1988. 